\title{
IMECE2005-80879
}

\section{THEORY, SIMULATION, AND HARDWARE: LAB DESIGN FOR AN INTEGRATED SYSTEM DYNAMICS EDUCATION}

\author{
Jonathan E. Clark \\ GRASP Laboratory \\ University of Pennsylvania \\ Philadelphia, Pennsylvania 19104 \\ Email: jonclark@grasp.upenn.edu
}

\author{
William R. Provancher \\ Department of Mechanical Engineering Department of Mechanical Engineering \\ University of Utah \\ Salt Lake City, Utah, $84112 \quad$ Stanford, California, 94305
}

\begin{abstract}
This article describes a series of experimental and simulation laboratory sessions that are designed to support a system dynamics course. A novel hardware setup is used in different configurations throughout the eight-lab sequence. The labs incrementally build upon themselves and culminate in the final lab with the stabilization of a coupled dynamic system. The labs augment the fundamental theory taught in class with hands-on experience using hardware and software simulations. We find that the labs that most effectively create and resolve tension between the results of physical experiments, simulation, and theory yield the most satisfying and effective lab sessions. The absence of any one of these three elements diminishes the educational value of the course. End-of-quarter lab surveys are used to quantify our findings.
\end{abstract}

\section{INTRODUCTION}

As engineering education seeks to adapt to the rapid advances in technology and effectively prepare students for an increasingly competitive 21 st century workforce, a number of alterations to the engineering curriculum are being considered. One area that has changed over the past two decades is the rise in the use of computer tools in the classroom. The training and use of CAD, CAM, FEA, and increasingly dynamic simulation software is now considered a core part of the curriculum. Simulation software has been incorporated into lectures, homework, and in many cases has become part of the laboratory aspect of the students education. As shown by $[1,2]$, the advantages of sim- ulation based assignments and labs include their flexibility, low cost, and versatility. In addition, their ability to allow students to quickly investigate design and parameter changes to complex and more 'real world' problems makes them an ideal addition to the standard curriculum.

With the increasing connectivity provided by the Internet, the possibility for, and interest in, distance learning has rapidly increased. The advantage of software labs is obvious in this context. On the other hand, there is still a strong motivation for exposing students to real systems with all of their nonlinearities and technical difficulties. Some innovative lab structures have been developed $[3,4]$, but these are still expensive, bulky, and difficult to maintain. As a consequence, a number of researchers, such as $[5,6]$ have sought ways, such as 'tele-labs', to extend the benefits of involving students in real, physical laboratory experiences to students in remote locations.

In addition to the research on simulation and hardware labs, there has been evidence that a series of goal-oriented labs that incrementally build upon themselves aids in the students' learning and increases their interest [7].

Our belief is that the best laboratory experiences combine software (simulation) and hardware. The rapid investigations allowed by simulation compliments the experience gained by dealing with the limitations of real, nonlinear, and noisy hardware systems. We developed a small, inexpensive, and reconfigurable hardware platform (described in the next section) that gives students a hands-on experience to compliment their simulation exercises. These platforms are used in a series of labs that are sequenced to build upon each other and culminate in a final control 
exercise.

This approach has arisen from our experience in a system dynamics course at Stanford University which has in the past used a variety of lab structures. In 1999 a haptics research-based approach [8] was taken, in 2000 a goal oriented [9] set of hardware labs were developed, and in 2001 a set of pure simulation labs were tried. The current labs were refined from previous years to maximize student learning and re-use of lab equipment, while minimizing cost and infrastructure complexity. The current sequence consists of eight 90 minutes labs. Each of the four lab stations accommodates 3-4 students which, over six lab periods, serves a class of about 70 students.

The labs cover first- and second-order dynamic systems, free and forced oscillations, motors, and an introduction to controls. Each pre-lab steps individual students through a Working Model ${ }^{\circledR}$ simulation of a related physical system. The students investigate the effect of changing model parameters on the system behavior. Then during each lab the students use the hardware to take physical measurements of the real system. Some of this was accomplished with hand measurements and some was done with an electronic data acquisition system. Post-lab questions then helped the students synthesize the principles learned in the software and hardware portions of the lab.

The hardware and electronics that were used throughout the lab sequence is described in the following section. The details of each lab are then described, including a description of how the hardware and software are adapted to convey the point of each lab. The paper then concludes with a discussion of the lab surveys results, some conclusions, and future directions for the development of the labs.

\section{HARDWARE DESCRIPTION}

One of the major difficulties in implementing a hardware based series of labs is the cost of establishing a physical infrastructure capable of demonstrating a wide variety of concepts. Our approach was to develop a single desktop platform that is reconfigured for each of the labs. Ultimately, the platform instantiates the classic controls problem of the inverted pendulum on a cart. Figure 1 shows the device in this configuration.

The $0.6 \mathrm{~m}$ long platform consists of a base upon which a cart is driven by a motor and capstan drive along the linear slide. The rotation of the pendulum is measured by a potentiometer and the motor has an 512 count HEDS 9100 encoder. Both of these connect to a PIC18F4431 microcontroller with built-in quadrature encoder inputs, 10-bit A/D converter, and RS232 output. The system also has a low cost 2-axis ADXL311 accelerometer $( \pm 2 g$ range) that is used in labs two, three, and four. The total cost for each lab station including the hardware, the motor, the sensors, and the electronics was less than $\$ 100$.

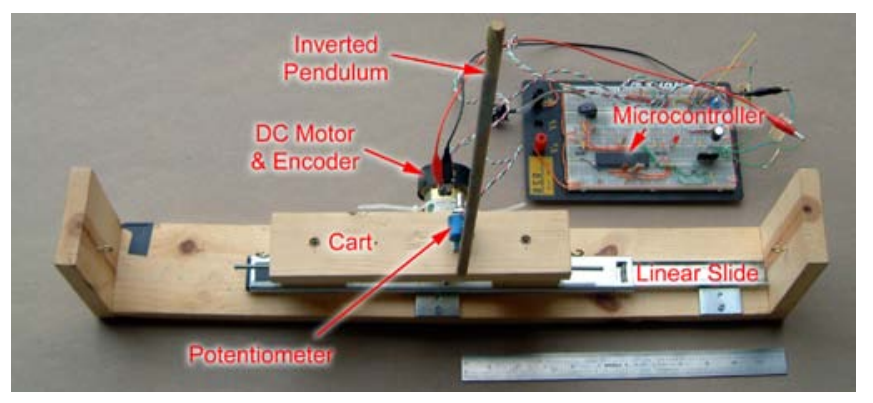

Figure 1. Hardware components for labs, assembled as an inverted pendulum on a cart. Also shown are the electronics and microcontroller.

\section{LAB OVERVIEW}

The labs were designed to complement the course lecture material beginning with first order differential equations and ending with linear feedback control of a multi-degree of freedom system. The first three labs have both simulation and hardware aspects, while labs 4 and 5 have only hardware and theoretical sections. Lab six is an open-ended project applying the principles learned in class and labs 1-5. In labs 7 and 8 the students are introduced to linear feedback control systems. The electronics for the last two labs was in development during the course, and consequently only the simulation portion of the lab was available to the students both years.

\section{LAB 1 - Motor Spindown}

In the first lab, as an example of a first-order dynamic system, the students examine the motion of motor-driven propeller spinning down to a stop. In the first pre-lab (due before the lab starts) the students are given an overview of Working Model ${ }^{\circledR}$ and are asked to use it to investigate the effect Coulomb and viscous friction on the spin-down profile of a propeller. Figure $2 \mathrm{a}$ shows characteristic spin down plots for a variety of parameter settings.

In the hardware portion of the first lab the students use the motor and pendulum (as shown in Fig. 2b) to conduct spin-down tests with a stop watch, and then compare their results to encoder readings. Data are taken with and without the attached 'propeller', and the rate of decay is recorded. The addition of the 'propeller' dramatically increases the mass and drag on the system. The shape of the decay curves is compared with the Working Model ${ }^{\circledR}$ simulation to estimate what type of friction dominates with and without the propeller attached to the motor. In the post-lab the students use the hardware data to estimate the magnitude of the motor's inertia and type and value of the damping. 

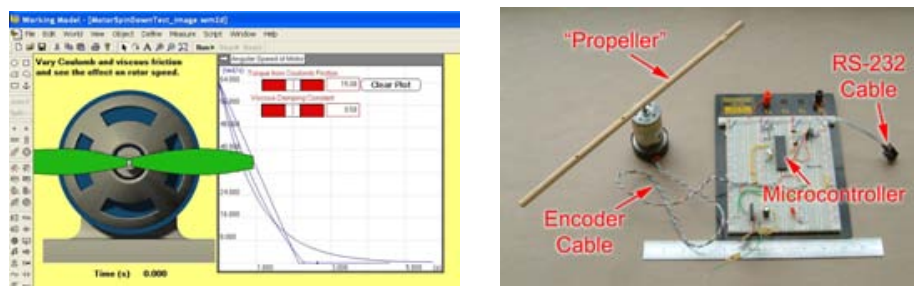

Figure 2. Simulation and hardware configuration for lab 1. (A) The Working Model ${ }^{\circledR}$ simulation showing spin down traces for viscous and Coulomb friction. (B) The motor and 'propeller' configured for lab 1.

\section{LAB 2 - Free Vibration (translational)}

Lab 2 introduces second-order dynamical systems. In Working Model ${ }^{\circledR}$, students investigate the effect of varying the natural frequency $\left(\omega_{n}\right)$ and damping ratio $(\zeta)$ on the behavior of a car's suspension. Students explore the response of this model to a step input when modified to give under- and over-damped behavior.

As shown in Fig. 3, an oscillatory system is created with the hardware by attaching the cart to the base via pre-stretched extension coil springs. The accelerometer (shown in the detail view within Fig. 3) measures the horizontal acceleration of the cart. The students use this data to experimentally determine the natural frequency and damping ratio of the system. They are asked to identify the primary potential sources of error. The experiment is then repeated by adding an object of known mass to the cart. This allows them in the post-lab to calculate the mass and damping of the cart, as direct measurement of these terms is not possible. Students learn that some physical parameters are easier to modify and measure than others, and that derived quantities such as the damping ratio are important from a practical standpoint.

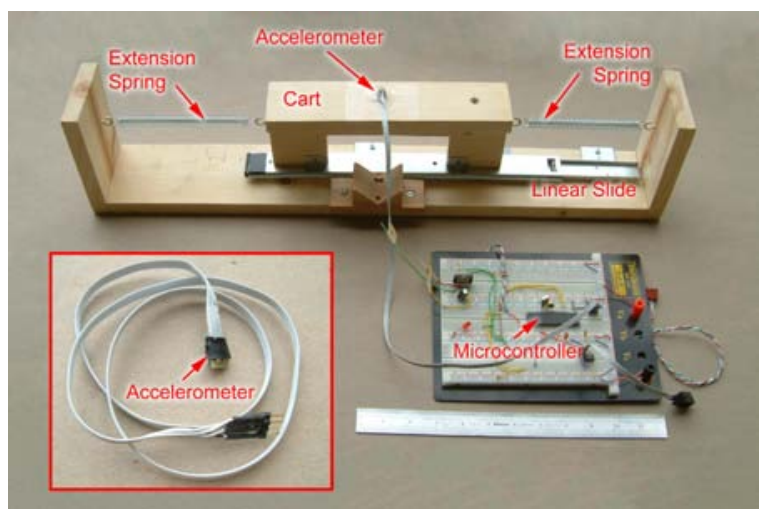

Figure 3. Hardware configuration for lab 2. The lateral motion of the cart is measured by the accelerometer shown in the insert.

\section{LAB 3 - Free Vibration (rotational)}

Lab 3 investigates a rotational second-order system, and reexamines natural frequency and decay. In the prelab, a Working Model $^{\circledR}$ simulation investigates how altering the location of the mass on a pendulum changes its frequency.

For the lab a 'guitar' shaped pendulum is chosen as it has a non-standard geometry. The students estimate and then measure the inertia using a bifilar pendulum [10]. The compliant flexure (shown in the insert of Fig. 4) gives an effective rotational stiffness and damping. The flexure and pendulum shape are taken from the author's research work in building dynamic running robots. Thus the students are not only exposed to the calculation and hand measurement of moment of inertia for a non-standard geometry, but also get a taste of how knowing these properties can be important in 'real' situations. In the post-lab, students perform a design exercise on the rotational system to meet a function specification based on the robot's locomotion dynamics.
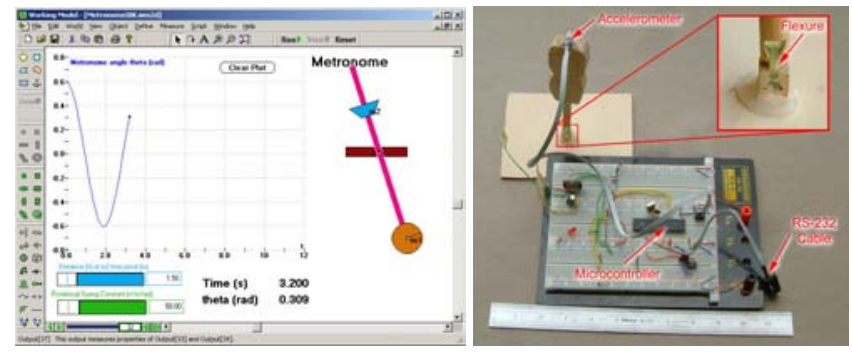

Figure 4. Simulation and hardware configuration for lab 3. (A) The Working Model ${ }^{\circledR}$ simulation of a metronome. (B) The spring-balanced inverted pendulum. The insert shows the compliant joint taken from a legged robot.

\section{LAB 4 - Forced Vibration}

Lab 4 examines how inhomogenous second-order ODEs model forced oscillations. For the pre-lab the students analytically solve a problem where an offset mass is driven by a motor on rickety platform. The students derive the expression for the steady-state acceleration of the platform as a function of the natural and driving frequencies.

For lab 4, an offset mass is added to the motor and the motor is directly mounted to the cart (rather than to the base as in labs 5, 7 and 8). As in lab 2, the addition of springs connecting the cart to the base specifies its natural frequency. The resulting system is shown in Fig. 5.

The students measure the damped natural frequency and damping ratio of the cart-spring system, and then use these values and the equations in the pre-lab to plot the predicted response of the system to a range of input frequencies. 


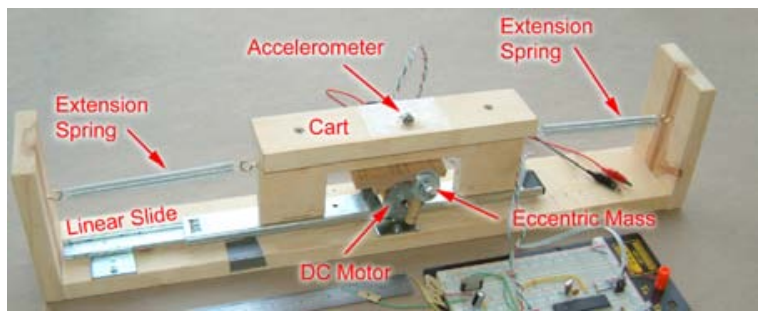

Figure 5. Hardware configuration for lab 4. The motor is attached to the cart, and the rotation of the mass offset from the motor's shaft induces an oscillatory force.

The motor is then driven at various frequencies using a variable voltage power supply and the period is checked via encoder readings. The resulting harmonic accelerations are measured and plotted against the analytical predictions. In the post-lab, students calculate the necessary parameter values in order to change the location of the resonate peak to fit within some functional requirements.

\section{LAB 5 - Motor Constant}

In lab 5 students determine the motor constant for their lab station by calculating the stall torque and no load speed. For this lab there is currently no simulation component. The resistance of the motor is measured with a multimeter and the no-load speed is calculated from encoder readings. The stall torque of the motor is determined by measuring the displacement of the cart driven against the resistance of a spring via the capstan, as shown in Fig. 6. The equations relating voltage and speed as well as torque and current are derived from the simplified motor diagram show at the bottom of Fig. 6. If the results of the two methods for determining the motor constant do not agree, a simple analysis is performed to identify the most likely source of error, and a second set of measurements is taken. This usually results in good agreement.

\section{LAB 6 - Open-ended group project}

Lab 6 is a team-based open-topic project lab. Each team submits 3 dynamic systems that they think might be interesting to study. During the pre-lab of the previous week the TAs help them select an appropriate topic (usually finding their proposals too complicated). As found in [11] having open-ended projects and labs as part of a class increases the students' interest in the subject matter and makes the value of the core material more apparent to them.

During the lab section, each team has an individual consultation with the TAs about how their project was going. Often the TAs make recommendations on appropriate simplifications, assist in the development of Working Model $^{\circledR}$ simulations, or help

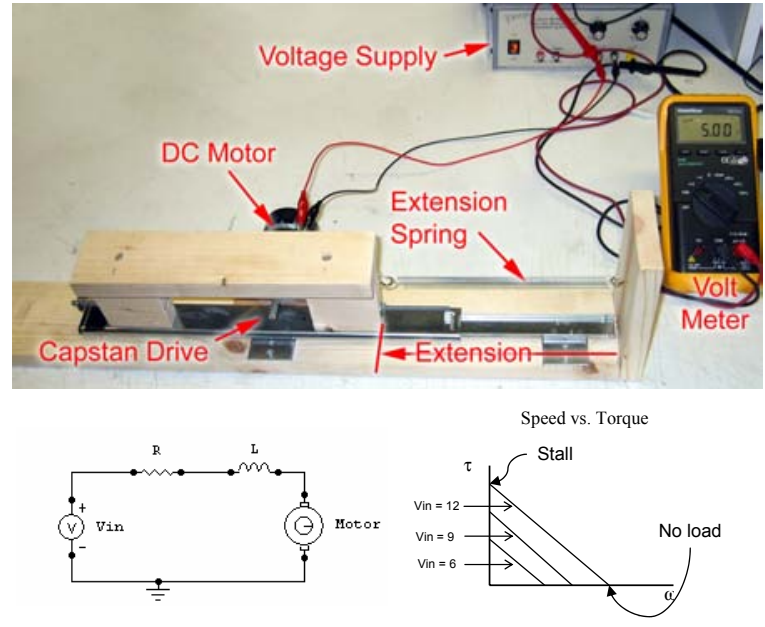

Figure 6. Hardware configuration and motor model for lab 5. (A) The motor is driven to stall conditions at $5 \mathrm{~V}$, stretching the spring and displacing the cart relative to the base. (B) The simplified motor diagram used to derive the expression used to calculate the motor constant.

with taking physical measurements. Each team submits a written report of the project including the project description, the model used, analytical results, and some interpretation of the results.
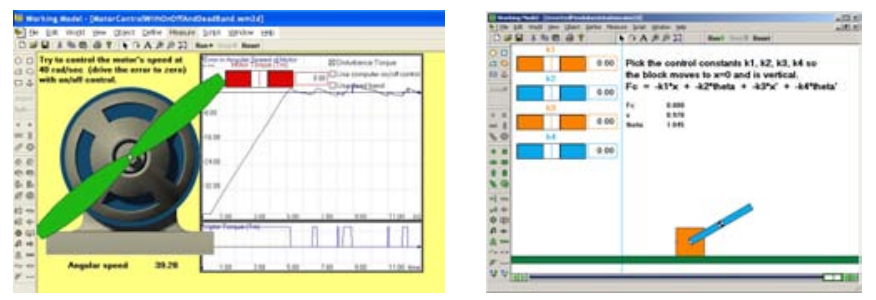

Figure 7. Simulation for labs 7 and 8. (A) Velocity control of a propeller with friction and bang-bang control and deadband (B) Gain selection for the control of an inverted pendulum on a cart.

\section{LAB 7 - Introduction to controls}

Lab 7 introduces bang-bang, deadband, and PI control. In the first year of implementing the labs, only the Working Model ${ }^{\circledR}$ portion of the lab was available, with students controlling the velocity of a spinning propeller with friction. Various control schemes and their impact on the error and the required input torque were measured. In the second year, the hardware was implemented, and the students used the motor to control the position of a nearly vertical pendulum. By adjusting the control parameters the students made an overdamped system into under- 
damped system. By adding integral control the students see how the steady-state error goes to zero, but how increasing the gains destabilizes the system.

\section{LAB 8 - Controls II, Inverted Pendulum}

In lab 8, students stabilize an inverted pendulum on a cart (currently in software only). The students are first asked to intuitively determine (through trial and error with Working Model ${ }^{\circledR}$ ) appropriate gains for stabilizing the cart and the pendulum. Some students found values to stabilize either the position of the cart or the pendulum independently, but all students found the trial-anderror approach to the coupled system fruitless. The analytically developed gains based on a related homework problem are then used to quickly stabilize the system, clearly demonstrating to the students the utility of the analytic framework developed in class.

When finished, the hardware portion of this lab will appear as shown in Fig. 1. We believe that adding the hardware to this lab will give a sense of completion to the labs and substantially increase their impact. The students will combine what they have learned in the early labs about the behavior and characteristics of the cart, the rotational pendulum, the motor, and the sensors with the theory learned in lecture in order to solve a non-trivial control problem. At this point the system is no longer a 'canned' or 'black box' setup, but a composition of discrete elements that they have become familiar with throughout the course.

\section{SURVEY RESULTS}

To evaluate the effectiveness of the labs, students were surveyed midway through the course and again at the end of the quarter. Students ranked each lab's effectiveness and perceived value, and provided comments or suggestions for improving them.

Personal, but non-identifiable, data for the students was taken in the mid-year review, covering the first four labs, in 2004. This included information about the students' expected grade in the class, lab group size, gender, preferred learning style, and which lab group they were in.

Each respondent ranked the perceived quality of each lab, the amount that it aided in their learning, how well the simulation and hardware segments were integrated into the lab, and the difficulty of the lab. They also recorded how much time they spent in the lab, as well as time spent on the pre- and post-lab sections, the difficulty of the write-ups, the length of the labs, and how much they contributed to the class.

Table 1 shows the overall ranking for 2003 and 2004, from 1 (worst) to 7 (best). On the whole, the labs were well received, with only labs 1,7 and 8 receiving noticeably lower marks. The sample size for 2003 was 16, and 58 for 2004.

Some interesting results from the more detailed data collected in 2004 includes the following:
Table 1. Rank of Lab Effectiveness

\begin{tabular}{clc}
\hline Lab \# & Description & Mean \\
\hline 1 & Motor Spindown & $5.02 \pm 0.87$ \\
2 & Free Vibration (Translational) & $5.54 \pm 0.71$ \\
3 & Free Vibration (Rotational) & $5.58 \pm 0.92$ \\
4 & Forced Vibration (Translational) & $5.53 \pm 1.23$ \\
5 & Motor Constant & $5.45 \pm 0.74$ \\
6 & Group Project & $5.40 \pm 1.13$ \\
7 & Introduction to Controls & $5.13 \pm 1.20$ \\
8 & Controls II & $5.13 \pm 1.06$ \\
\hline
\end{tabular}

- The labs were ranked equally well by students who expected to do well in the class (get an A- or better) as those who did not. The only exception was the lab writeups, which the 'A' students judged to be slightly easier.

- Females spent more time in the lab and thought the labs were of lower quality, but had a higher estimation of the labs' contribution to the class.

- The students in the first lab section of the week and the lab section that fell late on Friday afternoons had the lowest opinions of the labs.

- Lab groups with more than three students spent slightly less time in lab, but did not feel the labs were any less valuable than the class as a whole.

- There was no noticeable difference between seniors' and juniors' evaluations of the labs.

- The hardware scored slightly higher (5.3/7 vs. $4.9 / 7)$ than the simulation portion of the lab on its perceived contribution to the lab experience.

Of the seven learning style choices given, most of the students described their learning style as: Doing/action, logic/math, or visual. Students who described themselves as having either active or visual styles thought the overall contribution and quality of the labs was slightly higher than average, and the 'logic/math' learners thought that the labs were more difficult, of poorer quality, and did not aid in learning as much as the class average.

Students in 2003 were asked which labs were the most and least valuable to their overall learning. Lab 6 , the open-ended group project got the plurality of votes in both categories! The divergence of opinion was reflected in the comments written by the students. Some students loved the opportunity to apply what they 
had learned to a real system of interest. Comments such as "I loved this independent group project - challenging but my most favorite project of the quarter. We all cared about our project proposal." On the other hand some students have difficulty with the open-ended nature of the task as made clear by comments such as: "more guidance" or "Clarify the assignment. I felt like I never really knew what we were supposed to be doing."

\section{DISCUSSION OF RESULTS}

The unfamiliarity with the lab hardware and the corresponding logistical overhead may be to blame for the fact that the first lab received noticeably lower evaluations. The high marks for the remainder of the hardware labs suggests that there is a real advantage to using a consistent lab mechanism throughout the course.

The development of the labs is ongoing process. Evaluation of their impact at the current state where only some of the labs have both the simulation and the hardware components allows us to test our hypothesis that the combination of both is most desirable. Anecdotal evidence supports this, such as student comments like: "The Working Model portions were a great intro to the actual lab experiments" and "I think the [software and hardware] integrated well and it was neat to have a clean data set to compare the real behavior to."

More quantitatively, the survey results showed that the labs that ranked the highest in the student surveys were the ones that had the best integration of the hardware with simulation or analysis.

We believe that one reason that the combination of hardware and simulation is effective because these labs help the students see the benefits and limitations of each approach. Consequently, we feel that the simulation part of the early labs could be improved by explicitly modeling mechanical sources of error such as drift, bias, quantization, and noise and then comparing the results to physical system to evaluate the effect of filtering and other techniques to 'clean up' the data as has been done in [12].

Another reason that the labs with both hardware and software components are effective is that they appeal to a broader section of students. Particularly those students who describe themselves as visual or active/doing learners found that these labs contributed significantly to their educational experience.

\section{CONCLUSIONS AND FUTURE WORK}

The benefits of our unified hardware design include its costless than $\$ 100$ per station-and its ease of storage and deployment. Using the same platform for each lab also minimized the time spent familiarizing the students with the hardware, which in turn deceased their frustration and increased the amount of time they had to run experiments. Another aspect of the sequential labs, which we have not yet fully exploited, is that it allows for a goal-oriented set of labs. If at the beginning of the course the students are shown how in the final lab they will stabilize the inverted pendulum, then they will have some context for understanding how each of the labs brings them incrementally closer to this capability or goal.

One disadvantage of using this hardware is that students are only exposed to a single apparatus and set of behaviors. While the motor and encoder are not atypical of what students may find in later applications they certainly do not give them a wide sense of the scope of actuators they will encounter in their careers. On the other hand, great familiarity with this coupled second-order system gives the students a great grounding in dynamic systems. Furthermore, students were also exposed to a variety of 'real' systems during lecture demonstrations to show how their knowledge applies to other systems.

Having synthesized hardware, simulation, and basic theory, students are much better prepared to make decisions about which approach is most appropriate when faced with the open-ended engineering problems they face after leaving school. We believe that the labs described here are an excellent counterpart to the classroom instruction and that they significantly increase the mastery and retention of the core subject matter. Furthermore, the cost-effective nature of the lab hardware makes the wide dissemination of this laboratory sequence a real possibility. We believe that the wide adoption of this type of laboratory sequence would be of real benefit in preparing engineers for the challenges of the 21 st century.

\section{Appendix - Lab Kit - Bill of Materials}

Table 2. Bill of Materials

\begin{tabular}{clc}
\hline & Description & Cost \\
\hline 1 & Frame: $1 \times 4 \times 36$ "” pine board & $\$ 4$ \\
2 & Slider: drawer slide & $\$ 8$ \\
3 & Cart: $1 \times 4$ x 12", pine board & $\$ 1$ \\
4 & Motor/Encoder & $\$ 30$ \\
5 & Motor pulley (3/8", dia. alum rod) & $\$ 5$ \\
6 & Lok-tite (1 tube for all stations) & $\$ 5$ \\
7 & Extension springs (2 or 3 sets?) & $\$ 5$ \\
8 & Wood screws, \#4 and \#8 (1 box for all stations) & $\$ 3$ \\
9 & Breadboard, jumpers and connectors & $\$ 25$ \\
10 & PIC18F4431 Microcontroller & $\$ 8$ \\
\hline & & $\$ 94$
\end{tabular}




\section{REFERENCES}

[1] Cimbala, J., Moeykens, S., Kulkarni, A., and Parihar, A., 2004. "Using flowlab, a computational fluid dynamics tool, to facilitate the teaching of fluid mechanics". In Proceedings of ASME IMECE.

[2] Wankat, P., 2002. "Integrating the use of commercial simulators into lecture courses". Journal of Engineering Education, 91(1).

[3] Clark, W. W., and Hake, R., 1997. "Example of projectbased learning using a laboratory gantry-crane". In Proceedings of the 1997 27th Annual Conference on Frontiers in Education. Part 3 (of 3) Pittsburgh, PA, USA.

[4] Ghorbel, F., 1999. "Spherical pendulum system to teach key concepts in kinematics, dynamics, control, and simulation". IEEE Transactions on Education, 42(4).

[5] Casini, M., Prattichizzo, D., and Vicino, A., 2001. "The automatic control telelab: a remote control engineering laboratory". In Proceedins of the 40th Conference on Decision and Control, Orlando Florida USA.

[6] Shaheen, M., Loparo, K., and Buchner, M., 1998. "Remote laboratory experimentation". In Proc. American Control Conf., Philadelphia, PA.

[7] Lyons, J., Young, E., and Morehouse, J., 2001. "Developing a systems approach to engineering problem solving and design of experiments in a racecar-based laboratory course". Journal of Engineering Education, 90(1).

[8] Okamura, A., Richard, C., and Cutkosky, M., 2002. "Feeling is believing: Using a force-feedback joystick to teach dynamic systems". Journal of Engineering Education, 91(3), pp. 345-349.

[9] Cham, J., Stafford, B., and Cutkosky, M., 2001. "See labs run: A design-oriented laboratory for teaching dynamic systems". In Proceedings of ASME IMECE.

[10] Steidel, R. F., 1989. An introduction ot mechanical vibrations. Wiley, New York.

[11] Avitablile, P., Hodgkins, J., and Van Zandt, T., 2004. "Integrating fundamental stem material in a laboratory based dynamic systems course". In Proceedings of ASME IMECE.

[12] Avitablile, P., and Van Zandt, T., 2004. "Developing a virtual model of a second order system to simulation real laboratory measurement problems". In Proceedings of ASME IMECE.

\section{ACKNOWLEDGMENT}

We would like to thank Ryan Hanson, Arthur (Trey) McClung, and the other dedicated TAs who have brought these labs to life. 